\title{
UHRF1 promotes human osteosarcoma cell invasion by downregulating the expression of E-cadherin in an Rb1-dependent manner
}

\author{
WEI LIU ${ }^{1,2}$, RUI HONG QIAO ${ }^{1}$, DONG MING WANG ${ }^{1}$, XIAO WEI HUANG ${ }^{3}$, BING LI $^{1}$ and DONG WANG ${ }^{1}$ \\ ${ }^{1}$ Department of Orthopedics, Second Clinical Medical College, Shanxi Medical University, Taiyuan, Shanxi 215006; \\ ${ }^{2}$ Department of Orthopedics, The First People's Hospital of Jinzhong City of Shanxi Province, Jinzhong, Shanxi 030600; \\ ${ }^{3}$ Department of Gastroenterology, Tongji Medical College of Huazhong University of Science and Technology, \\ Wuhan, Hubei 226001, P.R. China
}

Received December 10, 2014; Accepted September 25, 2015

DOI: $10.3892 / \mathrm{mmr} .2015 .4515$

\begin{abstract}
Ubiquitin-like with plant homeodomain (PHD) and RING-finger domain 1 (UHRF1) maintains methylation patterns following DNA replication and is expressed at high levels in various types of human cancer. UHRF1 has been identified as a novel oncogene involved in the pathogenesis of hepatocellular carcinoma. Previous studies have demonstrated that inhibition of the expression of UHRF1 suppresses the proliferation of cancer cells. However, the role of UHRF1 in human osteosarcoma has not been investigated. The present study examined the expression levels of UHRF1 and retinoblastoma 1 (Rb1) in human osteosarcoma cell lines by western blot analysis. Stable overexpression of UHRF1 or knockdown of Rb1 was achieved by lentiviral transfection. Subsequently, a Cell Counting Kit-8 assay and a cell invasion assay were performed to detect the biological functions of UHRF1 in vitro. The results of the present study demonstrated that UHRF1 promoted the proliferation of human osteosarcoma cells. The present study also reported that UHRF1 was able to enhance the invasion of osteosarcoma cells in a retinoblastoma 1 (Rb1)-dependent manner. UHRF1 promoted invasion in Rb1-positive osteosarcoma cells, but not in Saos-2 cells with homozygous loss of Rb1. Similarly, knockdown of Rb1 in Rb1-positive osteosarcoma cells enhanced levels of invasion and eliminated the regulation of invasion by UHRF1. UHRF1 was found to inhibit the mRNA and protein expression levels of Rbl. Furthermore, deletion of $\mathrm{Rb} 1$ was found to suppress the expression of E-cadherin and
\end{abstract}

Correspondence to: Professor Dong Wang, Department of Orthopedics, Second Clinical Medical College, Shanxi Medical University, South Building, 382 Wuyi Road, Taiyuan, Shanxi 215006, P.R. China

E-mail: dong_wang123@126.com

Key words: ubiquitin-like with plant homeodomain and RING-finger domain 1, osteosarcoma, invasion, retinoblastoma 1, E-cadherin, epithelial-to-mesenchymal transition promote epithelial-to-mesenchymal transition (EMT). In addition, the overexpression of UHRF1 inhibited the expression of E-cadherin and promoted EMT via the suppression of Rb1. These data demonstrated that UHRF1 promotes osteosarcoma cell invasion by downregulating the expression of E-cadherin and increasing EMT in an Rb1-dependent manner.

\section{Introduction}

Osteosarcoma is the most frequent type of primary bone malignant tumor in children (1). It is considered to arise from malignant mesenchymal cells, which produce osteoid or immature bone (2). Although advanced treatment for osteosarcoma consists of aggressive adjuvant chemotherapy, the five-year survival rate of patients with high-grade osteosarcoma remains $<50 \%$ (3).

Ubiquitin-like with plant homeodomain (PHD) and RING finger domain 1 (UHRF1) is a multi-domain protein, which was initially identified as a nuclear protein associated with cell proliferation (4). As a human inverted CCAAT box-binding protein, UHRF1 is involved in regulating the expression of topoisomerase II $\alpha$ in proliferating cells (5). UHRF1 has been found to inhibit the mRNA and protein expression levels of Rb1 $(6,7)$. Deletion of Rb1 was found to suppress the expression of E-cadherin and promote epithelial-to-mesenchymal transition (EMT) (8). Furthermore, UHRF1 is involved in the methylation of newly synthesized $\mathrm{CpG}$ sequences during DNA replication $(9,10)$. UHRF1 binds preferentially to dimethylated and trimethylated histone 3 lysine 9 peptides, and this binding is required for the maintenance of DNA methylation (11). Through interaction with other nuclear proteins, including Tip60, histone deacetylase and G9A, UHRF1 may function as a bridge between DNA methylation and the histone methylation (12). Furthermore, significant overexpression of UHRF1 has been observed in several types of human tumor, including breast $(13,14)$, bladder (15), prostate (16) and lung cancer (17). The overexpression of UHRF1 has also been reported to reduce the radiosensitivity of human breast cancer cells and HeLa cells to $\gamma$-irradiation (18). Previous studies have demonstrated that UHRF1 is an oncogene in hepatocellular carcinoma $(19,20)$. 
Furthermore, the overexpression of UHRF1 destabilizes and delocalizes DNA (cytosine-5-)-methyltransferase 1 (DNMT1) and causes DNA hypomethylation in cancer cells (19). Despite these findings, little is known regarding the function of UHRF1 in human osteosarcoma cells. In the present study, the effects of UHRF1 on the invasion of osteosarcoma cells were examined, and the associated underlying mechanisms were investigated.

\section{Materials and methods}

Cell culture. MG-63, Saos-2, U2OS and HOS human osteosarcoma cells lines were obtained from American Type Culture Collection (Manassas, VA, USA). HEK293T cells were obtained from the Institute of Cell and Biochemistry Research of the Chinese Academy of Science (Shanghai, China). The cell lines were cultured with Dulbecco's modified Eagle's medium (DMEM; Invitrogen; Thermo Fisher Scientific, Inc., Waltham, MA, USA) supplemented with $10 \%$ fetal bovine serum (FBS; Invitrogen; Thermo Fisher Scientific, Inc.) in a humidified atmosphere containing $5 \% \mathrm{CO}_{2}$ at $37^{\circ} \mathrm{C}$.

Reagents. The mouse anti-human monoclonal antibodies targeting UHRF1 (cat no. ab57083; 1:2,000 dilution) and GAPDH (cat no. ab9484; 1:5,000 dilution) were purchased from Abcam (Cambridge, MA, USA). Human Rb1-specific rabbit polyclonal antibody (cat. no. 9313; 1:2,000 dilution) was purchased from Cell Signaling Technology, Inc. (Danvers, MA, USA). The mouse anti-human E-cadherin monoclonal antibody (cat no. sc-21791; 1:2,000 dilution) was obtained from Santa Cruz Biotechnology, Inc. (Dallas, TX, USA). The pLenti-UHRF1 lentiviral expression vector and the control vector were purchased from OriGene Technologies, Inc. (Beijing, China). PLKO.1 lentiviral vectors containing short hairpin (sh)RNA inserts against Rb1 were purchased from Sigma-Aldrich (St.Louis, MO,USA). The target sequences were as follows: shRb-A, 5'-GCCTTTGATTCGTTCCTTCTT-3' and shRb-B, 5'-TGTGAAATACTGGCCCGAGAA-3'.

Transfection and lentiviral transduction. Transfection of the cells was performed using FuGENE transfection reagent (Roche Diagnostics, Indianapolis, IN, USA), according to the manufacturer's protocol $\left(3.5 \times 10^{6}\right.$ cells in a $10 \mathrm{~cm}$ dish with $20 \mu \mathrm{l}$ FuGENE). The lentiviral expression vector containing the pLenti-UHRF1/PLKO.1 or the control/empty vector were transfected into the HEK293T cells. The recombinant lentivirus was subsequently harvested, filtered through Millipore Millex-HV $0.45 \mu \mathrm{M}$ polyvinylidene difluoride filters (Millipore, Billerica, MA, USA) and transduced into the target cells (MG-63, Saos-2 and U2OS cells at $60 \%$ confluence) with $8 \mu \mathrm{g} / \mathrm{ml}$ polybrene (Sigma-Aldrich). After $48 \mathrm{~h}$ of incubation, the cells were selected with fresh puromycin-containing media $(2.0 \mu \mathrm{g} / \mathrm{ml}$; Sigma-Aldrich). Following puromycin selection for $48 \mathrm{~h}$, the expression levels of UHRF1 and Rb1 were quantified using western blot analysis.

Cell Counting kit-8 (CCK-8) assay. Cell proliferation was determined using a CCK-8 assay (Dojindo, Kumamoto, Japan). Briefly, the cells were plated in 96-well plates at 2,500 cells/well and cultured in DMEM. Every $24 \mathrm{~h}, 10 \mu \mathrm{l} \mathrm{CCK}-8$ was added to each well containing $100 \mu 1$ DMEM once. The plates were then incubated for a further $2 \mathrm{~h}$ at $37^{\circ} \mathrm{C}$. The cell growth was monitored every $24 \mathrm{~h}$ for 7 days using the CCK- 8 assay. Absorbance was measured at $450 \mathrm{~nm}$ using a microplate reader (Infinite Pro 2000; Tecan GmbH, Grödig, Austria).

Cell invasion assay. To assess the role of UHRF1 in cell invasion, a total of $1 \times 10^{5}$ cells were suspended in $100 \mu 1$ DMEM supplemented with $10 \% \mathrm{FBS}$, and were seeded into the upper compartment of Matrigel-coated (BD Biosciences, Franklin Lakes, NJ, USA) Transwell chambers (24-well; $8 \mu \mathrm{m}$; Merck Millipore, Darmstadt, Germany). The cells were incubated for $24 \mathrm{~h}$ at $37^{\circ} \mathrm{C}$ in a $5 \% \mathrm{CO}_{2}$ chamber. The cells, which did not invade through the pores were removed using a cotton swab. The cells on the lower surface of the membrane were stained with a hematoxylin and eosin staining kit (Baixu of Biotechnology, Shanghai, China) and counted with a microscope (Leica DM 5000 B; Leica Microsystems, Wetzlar, Germany). Results are presented as the average number of cells in five randomly selected fields.

Western blot analysis. The cells were suspended in lysis buffer (Beyotime Institute of Biotechnology, Nanjing, China) containing a mixture of protease and phosphatase inhibitors (both from Roche Diagnostics GmbH, Mannheim, Germany). The cell lysates were then centrifuged at $11,500 \mathrm{x}$ g for $10 \mathrm{~min}$ at $4^{\circ} \mathrm{C}$. Protein concentrations were estimated using the Quick Start ${ }^{\mathrm{TM}}$ Bradford Protein Assay (Bio-Rad Laboratories, Inc., Hercules, CA, USA). The samples were separated by SDS-PAGE (4-29\% gradient; Bio-Rad Laboratories, Inc.) and then blotted onto a polyvinylidene difluoride membrane (Merck Millipore). The membranes were blocked with 5\% non-fat dried milk in Tris-buffered saline with 1\% Tween 20 (TBST; Sigma-Aldrich) for $1 \mathrm{~h}$ at room temperature prior to incubation with specific primary antibodies overnight at $4^{\circ} \mathrm{C}$. Following three washes with TBST, the membrane was incubated with peroxidase-conjugated secondary antibodies [anti-mouse immunoglobulin (Ig)G (cat no. 7076; 1:5,000 dilution) and anti-rabbit IgG (cat no. 7074) (both from Cell Signaling Technology, Inc.)] for $30 \mathrm{~min}$ at $37^{\circ} \mathrm{C}$ and then washed three times with TBST. The bound antibodies were detected using chemiluminescent horseradish peroxidase substrate (Merck Millipore) and images were captured using an LAS-4000 digital imaging system (GE Healthcare Life Sciences, Little Chalfont, UK).

Statistical analysis. Values are expressed as the mean \pm standard deviation. GraphPad 6.01 Prism software (GraphPad, Inc., La Jolla, CA, USA) was used for statistical analyses. Each experiment was repeated three times. $\mathrm{P}<0.05$ was considered to indicate a statistically significant difference.

\section{Results}

UHRF1 promotes the proliferation of human osteosarcoma cells. UHRF1 has been reported to be overexpressed in various types of cancer (13). The present study examined the expression levels of UHRF1 in four human osteosarcoma cell lines. As shown in Fig. 1A, the expression of UHRF1 was detected in all of the cell lines. Overexpression of UHRF1 can increase cell proliferation $(14,21)$, therefore, the effects of UHRF1 on the proliferation of human osteosarcoma cells were also 

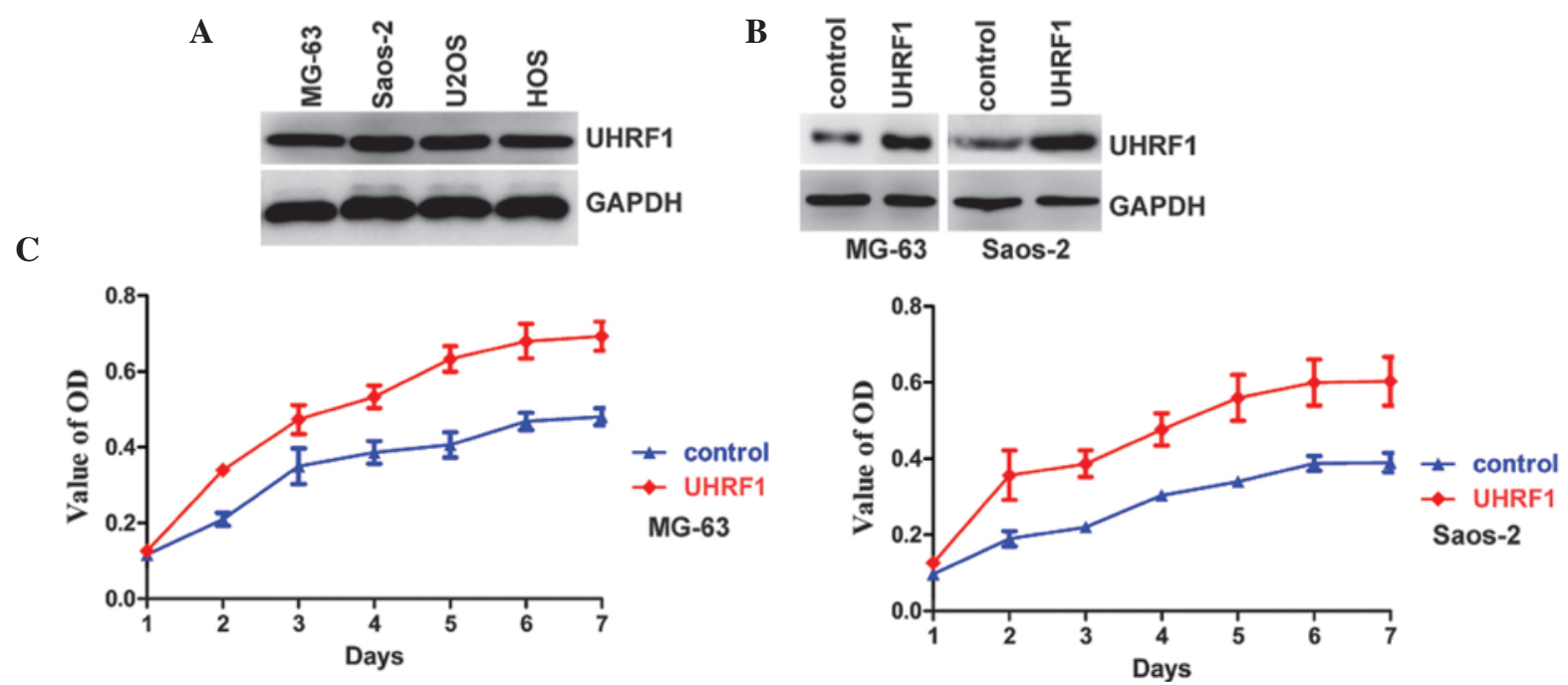

Figure 1. UHRF1 promotes the proliferation of human osteosarcoma cells. (A) Expression levels of UHRF1 and GAPDH were examined using western blot analysis in the MG-63, Saos-2, U2OS and HOS human osteosarcoma cells lines. (B) MG-32 and Saos-2 cells stably overexpressing UHRF1. Expression levels of UHRF1 and GAPDH were examined using western blot analysis. (C) Growth curves for MG-63 and Saos-2 cells stably expressing UHRF1 or the vector control. Data are presented as the mean \pm standard deviation. Cells overexpressing UHRF1 exhibited an increased proliferation rate $(\mathrm{P}<0.0001$, vs. control). UHRF1, ubiquitin-like with plant homeodomain and RING-finger domain 1; OD, optical density.
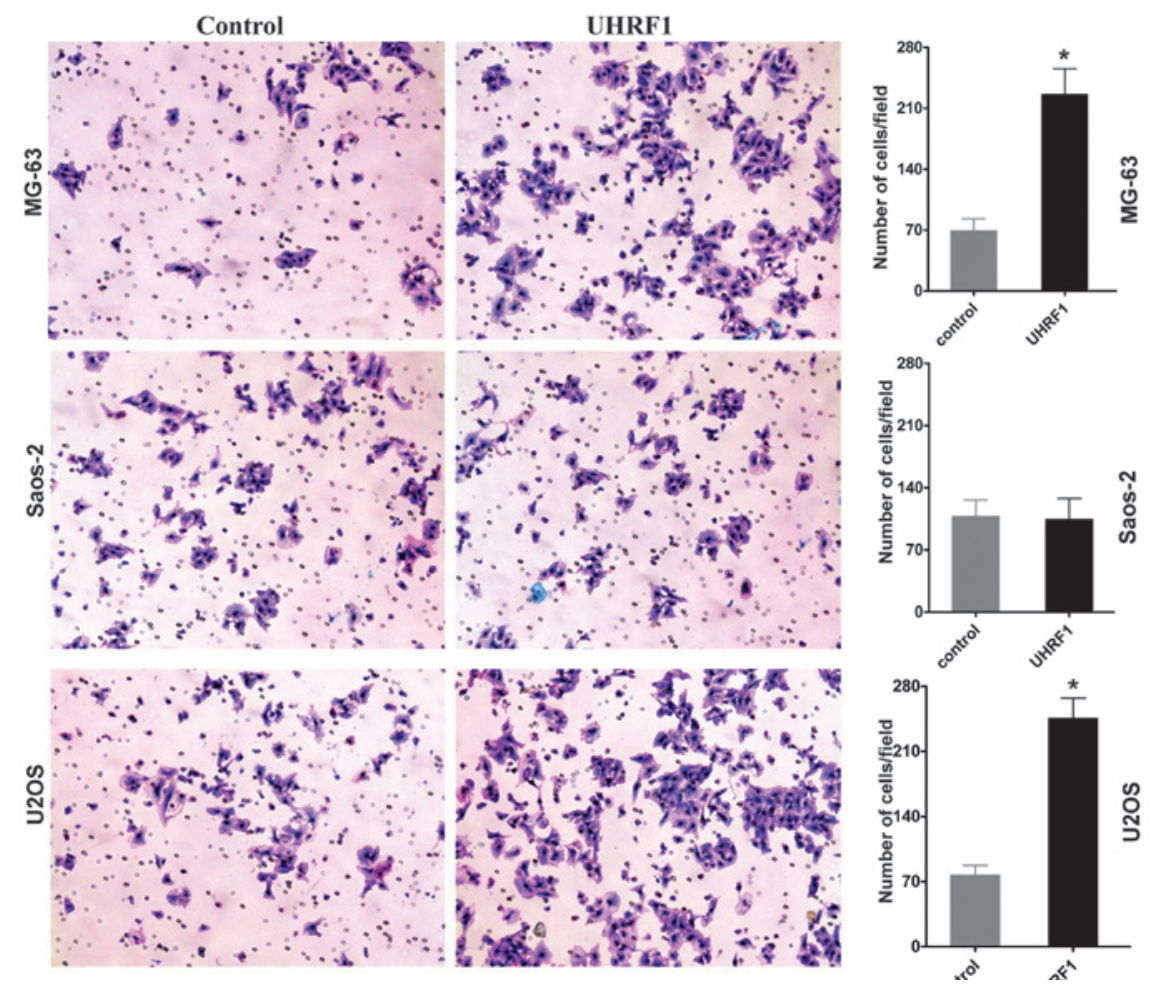

Figure 2. UHRF1 promotes the invasion of human osteosarcoma cells. MG-63, Saos-2 and U2OS cells stably expressing UHRF1 or transfected with the control vector. The invasion potential of these cells was determined using a Transwell assay. Representative images are shown on the left (magnification, $\mathrm{x} 40$ ), and quantification of five randomly-selected fields is shown on the right. Data are presented as the mean \pm standard deviation. " $\mathrm{P}<0.005$, vs. control. UHRF1, ubiquitin-like with plant homeodomain and RING-finger domain 1.

investigated. UHRF1 was stably overexpressed in the MG-63 and Saos-2 osteosarcoma cell lines (Fig. 1B). As expected, overexpression of UHRF1 increased the proliferation rate of the MG-63 and Saos-2 osteosarcoma cell lines (Fig. 1C).

UHRF1 promotes the invasion of MG-63 and U2OS human osteosarcoma cells, but not Saos-2 cells. In order to investigate the function of UHRF1 on the invasion of osteosarcoma cells, Transwell invasion assays were used with three human osteosarcoma cell lines in vitro. The overexpression of UHRF1 significantly increased the invasion of MG-63 and U2OS human osteosarcoma cells (Fig. 2). By contrast, the overexpression of UHRF1 had no significant effect on the invasion of the Saos-2 cells (Fig. 2). Homozygous deletion of the Rb1 gene has been 
A

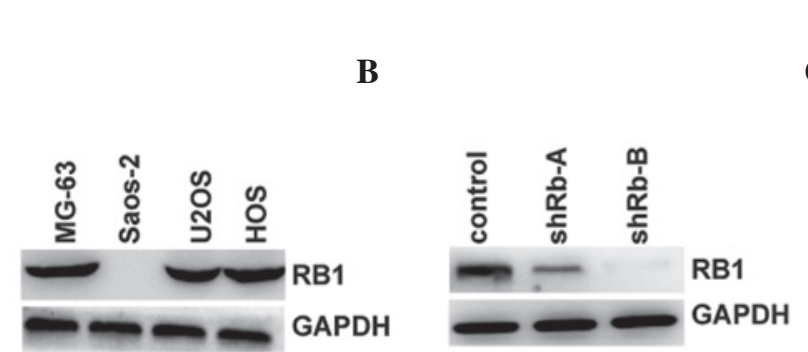

C



D
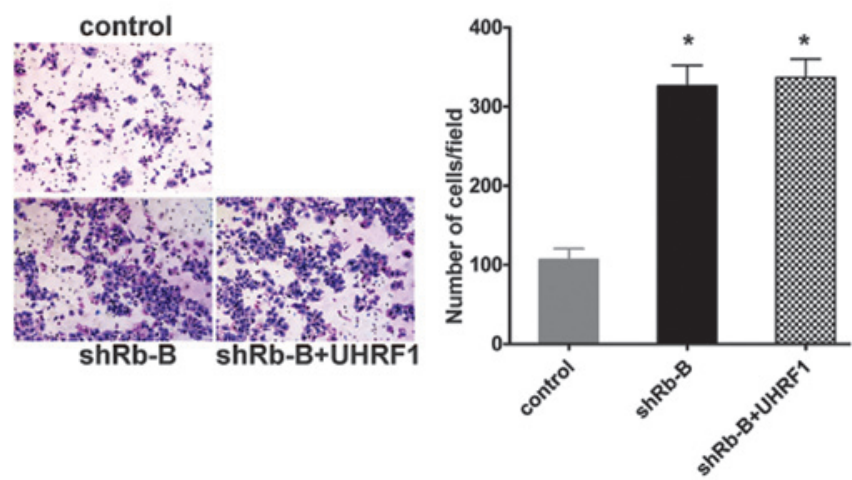

Figure 3. UHRF1 promotes the invasion of human osteosarcoma cells in an Rb1-dependent manner. (A) Expression levels of Rb1 and GAPDH were examined using western blot analysis in the MG-63, Saos-2, U2OS and HOS human osteosarcoma cells lines. (B) MG-63 cells were transfected with Rb1-shRNA (shRb-A and shRb-B) or negative control, and the expression levels of Rb1 and GAPDH were examined using western blot analysis. (C) UHRF1 was overexpressed in the Rb1 stable knockdown (shRb-B) MG-63 cells. The expression levels of UHRF1, Rb1 and GAPDH were examined using western blot analysis. (D) MG-63 cells were transfected with negative control (control) or Rb1-shRNA (shRb-B). Overexpression of UHRF1 was observed in the Rb1-shRNA MG-63 cells (shRb-B + UHRF1). The invasion potential of these cells was determined using a Transwell assay. Representative images are shown on the left (magnification, $\mathrm{x} 40$ ), and quantification of five randomly-selected fields is shown on the right. Data are presented as the mean \pm standard deviation. ${ }^{*} \mathrm{P}<0.0002$, vs. control. UHRF1, ubiquitin-like with plant homeodomain and RING-finger domain 1; Rb1, retinoblastoma 1; shRNA, short hairpin RNA.

identified in Saos-2 cells $(22,23)$, whereas MG-63 and U2OS cells exhibit normal expression levels of Rb1 $(23,24)$. Therefore, the present study hypothesized that $\mathrm{Rb} 1$ may be involved in regulating the invasion of osteosarcoma cells by UHRF1.

UHRF1 promotes the invasion of human osteosarcoma cells in an Rb1-dependent manner. To test the hypothesis that Rb1 may be involved in the regulation of the invasion of osteosarcoma cells by UHRF, the expression levels of Rb1 were quantified in the osteosarcoma cell lines. As expected, the MG-63 and U2OS cells exhibited normal expression levels of Rbl. The expression of $\mathrm{Rb} 1$ in the Saos-2 cells containing the homozygous deletion of Rb1 was not detected (Fig. 3A). To further investigate the mechanism underlying the effect of UHRF1 on the regulation of osteosarcoma cell invasion, the expression of Rb1 was stably knocked down in the MG-63 cells (Fig. 3B). The stable knowndown of Rb1 resulted in UHRF1 being overexpressed in the MG-63 cells (Fig. 3C). Following the knockdown of Rb1 in the Rb1-positive MG-63 cells, the invasion of the cells increased. By contrast, the indiced overexpression of UHRF1 had no effect on the invasion of the MG-63 cells following stable Rbl knockdown (Fig. 3D). These results indicated that Rbl appeared to be responsible for the regulation of cell invasion by UHRF1.

UHRF1 suppresses the expression of E-cadherin and promotes epithelial-mesenchymal transition (EMT) through the inhibition of Rb1. UHRF1 can inhibit the expression levels of Rb1 at
A

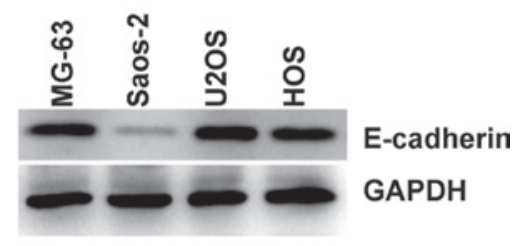

B

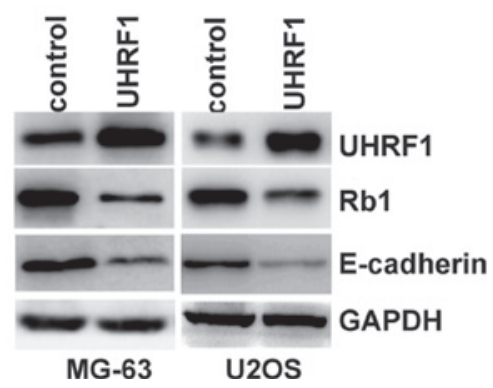

Figure 4. UHRF1 inhibits the expression of E-cadherin. (A) Expression levels of E-cadherin and GAPDH were examined using western blotting in the MG-63, Saos-2, U2OS and HOS human osteosarcoma cell lines. (B) UHRF1 was overexpressed in the MG-63 and U2OS cells. Expression levels of UHRF1, Rb1, E-cadherin and GAPDH were examined using western blotting. UHRF1, ubiquitin-like with PHD and RING-finger domain 1; Rb1, retinoblastoma 1.

the protein and mRNA levels (6). Furthermore, the inhibition of Rb1 suppresses the expression of E-cadherin and increases EMT (8). The present study hypothesized that UHRF1 may inhibit the expression of Rb1 and thereby suppress the expression 


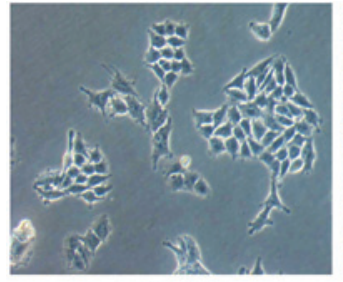

control

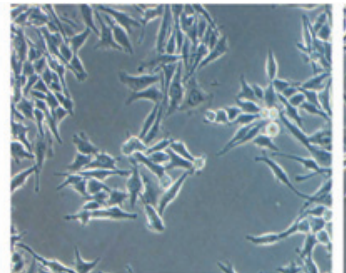

UHRF1

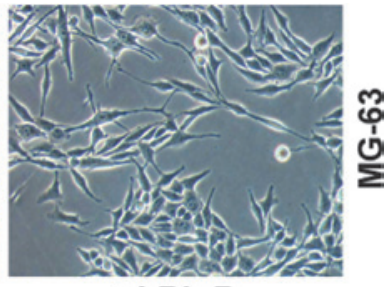

shRb-B

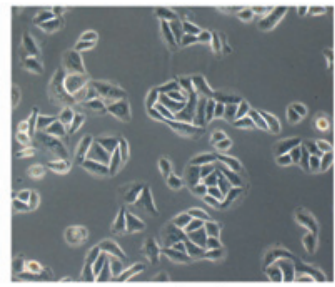

control



UHRF1

Figure 5. UHRF1 promotes epithelial-mesenchymal transition. Top row, morphological changes induced by overexpression of UHRF1 or knockdown of retinoblastoma 1 in MG-63 cells; lower row, morphological changes induced by overexpression of UHRF1 in Saos-2 cells. Magnification, x100. UHRF1, ubiquitin-like with plant homeodomain and RING-finger domain 1; sh, short hairpin RNA.

of E-cadherin. E-cadherin is known to suppress the invasion of cancer cells (25). Therefore, the UHRF1-mediated promotion of invasion may be the result of E-cadherin inhibition. The results of the present study demonstrated that the expression levels of E-cadherin were significantly lower in the Saos-2 cells, compared with the other Rb1-positive cells (Fig. 4A). Furthermore, overexpression of UHRF1 in the MG-63 and U2OS cells inhibited the expression of Rb1 and E-cadherin (Fig. 4B). The loss of E-cadherin is the initial or primary cause for EMT (26). In the present study, the UHRF1-overexpressing MG-63 cells exhibited marked changes in cell morphology, with transformation of the cobblestone-like epithelial cells to an elongated fibroblast-like morphology, and with pronounced cellular scattering that indicated EMT (Fig. 5). Similarly, knockdown of Rb1 in the MG-63 cells also showed EMT characteristic patterns (Fig. 5). However, UHRF1 had no effect on the cell morphology of the Saos-2 cells with the Rb1 deletion (Fig. 5). These results further supported the hypothesis that UHRF1 inhibits the expression of E-cadherin and promotes EMT through the suppression of Rb1.

\section{Discussion}

Osteosarcoma arises from malignant mesenchymal cells, which produce osteoid or immature bone, and is the most frequent type of primary bone malignant tumor (27). Despite advanced treatment for osteosarcoma, which combines chemotherapy, surgery and occasionally radiotherapy, the five-year survival rate for patients with high-grade osteosarcoma remains $<50 \%$ (3). Therefore, novel targeted molecular therapeutic techniques for osteosarcoma are required.

UHRF1, also known as inverted CCAAT box-binding protein of $90 \mathrm{kDa}$, contains different domains, including an E3-ligase RING domain, a SET and RING-associated (SRA) domain, a PHD finger domain and a tandem tudor domain (28). The SRA domain of UHRF1 specifically binds to hemi-methylated $\mathrm{CpG}$ following DNA replication, and recruits DNMT1 to methylate the newly synthesized DNA strand (29). UHRF1 is overexpressed and associated with tumor stages, and predicts poor prognosis in various types of cancer $(15,21)$. UHRF1 has previously been identified as an oncogene in hepatocellular carcinoma $(19,20)$. Furthermore, the overexpression of UHRF1 destabilizes and delocalizes DNMT1, and causes DNA hypomethylation in cancer cells (19). Although several studies have demonstrated the function of UHRF1 in tumorigenesis and tumor progression $(14,17,20)$, little is known regarding the function of UHRF1 in human osteosarcoma cells. In the present study, the expression of UHRF1 was detected in all of the human osteosarcoma cell lines examined. Furthermore, the overexpression of UHRF1 increased the proliferation of rates of the osteosarcoma cell lines.

EMT, during which epithelial cells are transdifferentiated to a mesenchymal state, is considered to be important in the initiation of the invasion and metastasis of cancer cells (30,31). Loss of E-cadherin is considered to be the most fundamental event during EMT (32). Deregulation in the expression of several genes or microRNAs has been reported to downregulate the expression of E-cadherin $(33,34)$. As an oncogene, UHRF1 is able to bind to methylated DNA and recruit transcriptional repressors to suppress the transcription of several tumor suppressor genes $(35,36)$. Therefore, UHRF1 may regulate the transcription of E-cadherin. The deletion of Rb1 is associated with downregulation of the expression of E-cadherin and increased EMT (8). UHRF1 binds to the Rb1 gene promoter and inhibits the expression of Rb1 (6). The results of the present study demonstrated that UHRF1 promoted the invasion of osteosarcoma cells of the MG-63 and U2OS cell lines, but not of the Saos-2 cell line. Saos-2 cells undergo homozygous deletion of the Rb1 gene $(22,23)$, therefore the present study hypothesized that Rb1 may be involved in the regulation of the invasion of osteosarcoma cells by UHRF1. Further investigations demonstrated that knockdown of the expression of Rb1 in the Rb1-positive cells eliminated the regulation of invasion by UHRF1. Furthermore, the expression levels of E-cadherin were consistent with the Rb1 status. The results of the present study also demonstrated that the overexpression of UHRF1 significantly downregulated the expression levels of Rb1 and E-cadherin in the Rb1-positive cells. Similarly, overexpression of UHRF1 in the MG-63 cells 
resulted in marked changes in cell morphology, indicating EMT, although this was not observed in the Saos-2 cells. The knockdown of Rbl led to the observation of similar changes in EMT in the UHRF1-overexpressing MG-63 cells.

In conclusion, the present study revealed that UHRF1 promoted human osteosarcoma cell invasion by downregulating the expression of E-cadherin and increasing EMT, in an Rb1-dependent manner.

\section{Acknowledgements}

The present study was supported by grants from the Science and Technology Planning Project of Jinzhong City (grant. no. N1312) and from the National Natural Science Foundations of China (grant. no. 81100293).

\section{References}

1. Goguet-Surmenian E, Richard-Fiardo P, Guillemot E, Benchetrit M, Gomez-Brouchet A, Buzzo P, Karimdjee-Soilihi B, Alemanno P, Michiels JF, Schmid-Alliana A and Schmid-Antomarchi H: CXCR7-mediated progression of osteosarcoma in the lungs. Br J Cancer 109: 1579-1585, 2013.

2. Kansara M, Teng MW, Smyth MJ and Thomas DM: Translational biology of osteosarcoma. Nat Rev Cancer 14: 722-735, 2014.

3. Liu Y, Wu Y, Gu S, Sun Z, Rui Y, Wang J, Lu Y, Li H, Xu K and Sheng P: Prognostic role of CD44 expression in osteosarcoma: Evidence from six studies. Diagn Pathol 9: 140, 2014.

4. Chu J, Loughlin EA, Gaur NA, SenBanerjee S, Jacob V, Monson C, Kent B, Oranu A, Ding Y, Ukomadu C and Sadler KC: UHRF1 phosphorylation by cyclin A2/cyclin-dependent kinase 2 is required for zebrafish embryogenesis. Mol Biol Cell 23: 59-70, 2012.

5. Hopfner R, Mousli M, Jeltsch JM, Voulgaris A, Lutz Y, Marin C, Bellocq JP, Oudet P and Bronner C: ICBP90, a novel human CCAAT binding protein, involved in the regulation of topoisomerase IIalpha expression. Cancer Res 60: 121-128, 2000.

6. Jeanblanc M, Mousli M, Hopfner R, Bathami K, Martinet N, Abbady AQ, Siffert JC, Mathieu E, Muller CD and Bronner C: The retinoblastoma gene and its product are targeted by ICBP90: A key mechanism in the G1/S transition during the cell cycle. Oncogene 24: 7337-7345, 2005.

7. Benavente CA, Finkelstein D, Johnson DA, Marine JC, Ashery-Padan R and Dyer MA: Chromatin remodelers HELLS and UHRF1 mediate the epigenetic deregulation of genes that drive retinoblastoma tumor progression. Oncotarget 5: 9594-9608, 2014.

8. Arima Y, Inoue Y, Shibata T, Hayashi H, Nagano O, Saya H and Taya $\mathrm{Y}: \mathrm{Rb}$ depletion results in deregulation of E-cadherin and induction of cellular phenotypic changes that are characteristic of the epithelial-to-mesenchymal transition. Cancer Res 68: 5104-5112, 2008.

9. Arita K, Ariyoshi M, Tochio H, Nakamura Y and Shirakawa M: Recognition of hemi-methylated DNA by the SRA protein UHRF1 by a base-flipping mechanism. Nature 455: 818-821, 2008.

10. Hashimoto H, Vertino PM and Cheng X: Molecular coupling of DNA methylation and histone methylation. Epigenomics 2: 657-669, 2010.

11. Rothbart SB, Krajewski K, Nady N, Tempel W, Xue S, Badeaux AI, Barsyte-Lovejoy D, Martinez JY, Bedford MT, Fuchs SM, et al: Association of UHRF1 with methylated H3K9 directs the maintenance of DNA methylation. Nat Struct Mol Biol 19: 1155-1160, 2012.

12. Unoki M: Current and potential anticancer drugs targeting members of the UHRF1 complex including epigenetic modifiers. Recent Pat Anticancer Drug Discov 6: 116-130, 2011.

13. Alhosin M, Sharif T, Mousli M, Etienne-Selloum N, Fuhrmann G, Schini-Kerth VB and Bronner C: Down-regulation of UHRF1, associated with re-expression of tumor suppressor genes, is a common feature of natural compounds exhibiting anti-cancer properties. J Exp Clin Cancer Res 30: 41, 2011.

14. Li XL, Xu JH, Nie JH and Fan SJ: Exogenous expression of UHRF1 promotes proliferation and metastasis of breast cancer cells. Oncol Rep 28: 375-383, 2012.

15. Zhang Y, Huang Z, Zhu Z, et al: Upregulated UHRF1 promotes bladder cancer cell invasion by epigenetic silencing of KiSS1. PLoS One 9: e104252, 2014.
16. Babbio F, Pistore $\mathrm{C}$, Curti L, et al: The SRA protein UHRF1 promotes epigenetic crosstalks and is involved in prostate cancer progression. Oncogene 31: 4878-4887, 2012.

17. Daskalos A, Oleksiewicz U, Filia A, et al: UHRF1-mediated tumor suppressor gene inactivation in nonsmall cell lung cancer. Cancer 117: 1027-1037, 2011.

18. Li XL, Meng QH and Fan SJ: Adenovirus-mediated expression of UHRF1 reduces the radiosensitivity of cervical cancer HeLa cells to gamma-irradiation. Acta Pharmacol Sin 30: 458-466, 2009.

19. Mudbhary R, Hoshida Y, Chernyavskaya Y, Jacob V, Villanueva A, Fiel MI, Chen X, Kojima K, Thung S, Bronson RT, et al: UHRF1 overexpression drives DNA hypomethylation and hepatocellular carcinoma. Cancer Cell 25: 196-209, 2014.

20. Zhuo H, Tang J, Lin Z, et al: The aberrant expression of MEG3 regulated by UHRF1 predicts the prognosis of hepatocellular carcinoma. Mol Carcinog: Jan 16, 2015 (Epub ahead of print).

21. Zhou L, Zhao X, Han Y, Lu Y, Shang Y, Liu C, Li T, Jin Z, Fan D and Wu K: Regulation of UHRF1 by miR-146a/b modulates gastric cancer invasion and metastasis. FASEB J 27: 4929-4939, 2013.

22. Manning AL, Yazinski SA, Nicolay B, Bryll A, Zou L and Dyson NJ: Suppression of genome instability in pRB-deficient cells by enhancement of chromosome cohesion. Mol Cell 53: 993-1004, 2014.

23. Rosemann M, Gonzalez-Vasconcellos I, Domke T, Kuosaite V, Schneider R, Kremer M, Favor J, Nathrath M and Atkinson MJ: A Rb1 promoter variant with reduced activity contributes to osteosarcoma susceptibility in irradiated mice. Mol Cancer 13: 182, 2014.

24. Ory B, Blanchard F, Battaglia S, Gouin F, Rédini F and Heymann D: Zoledronic acid activates the DNA S-phase checkpoint and induces osteosarcoma cell death characterized by apoptosis-inducing factor and endonuclease-G translocation independently of p53 and retinoblastoma status. Mol Pharmacol 71: 333-343, 2007.

25. Lau MT, Klausen C and Leung PC: E-cadherin inhibits tumor cell growth by suppressing PI3K/Akt signaling via $\beta$-catenin-Egr1-mediated PTEN expression. Oncogene 30: 2753-2766, 2011

26. Lombaerts M, van Wezel T, Philippo K, Dierssen JW, Zimmerman RM, Oosting J, van Eijk R, Eilers PH, van de Water B, Cornelisse CJ and Cleton-Jansen AM: E-cadherin transcriptional downregulation by promoter methylation but not mutation is related to epithelial-to-mesenchymal transition in breast cancer cell lines. Br J Cancer 94: 661-671, 2006.

27. Cao ZQ, Shen Z and Huang WY: MicroRNA-802 promotes osteosarcoma cell proliferation by targeting p27. Asian Pac J Cancer Prev 14: 7081-7084, 2013.

28. Cheng J, Yang Y, Fang J, Xiao J, Zhu T, Chen F, Wang P, Li Z, Yang $\mathrm{H}$ and $\mathrm{Xu}$ Y: Structural insight into coordinated recognition of trimethylated histone $\mathrm{H} 3$ lysine 9 (H3K9me3) by the plant homeodomain (PHD) and tandem tudor domain (TTD) of UHRF1 (ubiquitin-like, containing PHD and RING finger domains, 1) protein. J Biol Chem 288: 1329-1339, 2013.

29. Sharif J, Muto M, Takebayashi S, Suetake I, Iwamatsu A, Endo TA, Shinga J, Mizutani-Koseki Y, Toyoda T, Okamura K, et al: The SRA protein Np95 mediates epigenetic inheritance by recruiting Dnmt1 to methylated DNA. Nature 450: 908-912, 2007.

30. Puisieux A, Brabletz T and Caramel J: Oncogenic roles of EMT-inducing transcription factors. Nat Cell Biol 16: 488-494, 2014.

31. Kalluri R and Weinberg RA: The basics of epithelial-mesenchymal transition. J Clin Invest 119: 1420-1428, 2009.

32. Xiong H, Hong J, Du W, Lin YW, Ren LL, Wang YC, Su WY, Wang JL, Cui Y, Wang ZH and Fang JY: Roles of STAT3 and ZEB1 proteins in E-cadherin down-regulation and human colorectal cancer epithelial-mesenchymal transition. J Biol Chem 287: 5819-5832, 2012.

33. Sun XJ, Liu H, Zhang P, Zhang XD, Jiang ZW and Jiang CC: miR-10b promotes migration and invasion in nasopharyngeal carcinoma cells. Asian Pac J Cancer Prev 14: 5533-5537, 2013.

34. Wang YP, Wang MZ, Luo YR, Shen Y and Wei ZX: Lentivirus-mediated shRNA interference targeting SLUG inhibits lung cancer growth and metastasis. Asian Pac J Cancer Prev 13: 4947-4951, 2012.

35. Kim JK, Estève PO, Jacobsen SE and Pradhan S: UHRF1 binds G9a and participates in p21 transcriptional regulation in mammalian cells. Nucleic Acids Res 37: 493-505, 2009.

36. Guan D, Factor D, Liu Y, Wang Z and Kao HY: The epigenetic regulator UHRF1 promotes ubiquitination-mediated degradation of the tumor-suppressor protein promyelocytic leukemia protein. Oncogene 32: 3819-3828, 2013. 